\title{
Combined Heat, Air, Moisture and Pollutant Simulations (CHAMPS) research for building and urban energy efficiency and environmental quality analysis
}

\author{
Zhi Gao', Weihui Liang', Shanshan Shi', Jianshun Zhang ${ }^{1,2}$ (ه) \\ (Guest Editors) \\ 1. School of Architecture and Urban Planning, Nanjing University, 22 Hankou Road, Nanjing, Jiangsu Province, 210093, China \\ 2. Department of Mechanical and Aerospace Engineering, Syracuse University, 263 Link Hall, Syracuse, NY 13244, USA
}

๑) Tsinghua University Press and Springer-Verlag GmbH Germany, part of Springer Nature 2021

The energy and environmental performance of buildings and communities depends on how the heat, air, moisture and pollutant flows are managed and controlled in the system. Combined Heat, Air, Moisture and Pollutant Simulations (CHAMPS) research and development is vital to the optimal design and operation of building and urban energy and environmental systems. The $16^{\text {th }}$ International Forum and Workshop on Combined Heat, Air, Moisture and Pollutant Simulations (CHAMPS 2019) returned to Nanjing University, China on October 20-22, 2019. Previously, Nanjing University held the $8^{\text {th }}$ forum and workshop in 2011 and a topical issue was published on Building Simulation journal in December 2011 (Volume 4, Number 4).

CHAMPS 2019 covered research on multiple scales ranging from materials and microenvironment around a person to buildings and their surrounding urban environments. The papers published in this topical issue include selected papers presented at the forum and additional papers submitted directly to the journal. All papers went through the rigorous peer review process of the journal. A brief overview of each paper is given below:

- The paper by Shen et al. (2021) developed a new modularbased Green Design Studio (GDS) platform for fast and accurate performance analysis for early stage green building design. A simplified physics-based model, the ResistanceCapacitance (RC) model was proposed as a generic simulation model, which is significantly faster than conventional simulation tools such as EnergyPlus, and hence more practical for use in real-time design interaction and optimization. The proposed GDS approach has the potential to provide a fast and reliable feedback on performance prediction and makes it easier to modify

E-mail: jszhang@syr.edu

- the building design and evaluate the performance of each "green" feature in near-real time in design.

- The paper by Liu et al. (2021) developed a procedure to estimate the parameters of mechanistic volatile organic compound (VOC) emission source models from chamber testing data. Results indicated that estimates converged to the original parameter values used for the data generation and the error of estimated parameters $D_{\mathrm{m}}$ (in-material diffusion coefficient), $C_{\mathrm{m} 0}$ (initial concentration) and $K_{\mathrm{ma}}$ (partition coefficient) were within $\pm 10 \%, \pm 23 \%$, and $\pm 25 \%$ of the true values, respectively. The adoption of data analysis would result in a database of mechanistic model parameters for evaluating the impact of material emissions on indoor pollution load and indoor air quality (IAQ).

- The paper by Zhao et al. (2021) developed a probabilisticbased method to evaluate the hygrothermal performance of building components. The Latin hypercube sampling technique, incorporated with the correlation structure among the inputs, was applied to generate random samples that follow the intrinsic relations. The performance of an internally insulated masonry wall was evaluated by applying the proposed approach against different criteria. The method can provide a comprehensive risk analysis and support the decision-maker and engineer in the design and optimization of building components.

- The paper by Zhou et al. (2021b) proposed an action-based Markov chain modeling approach to predict the window operating behavior in office spaces. They found that this approach can simulate occupants' window operating behavior dynamically within the building and heating, ventilation, and air conditioning (HVAC) domains effectively. Compared with the state-based Markov chain 
modeling approach, the results of this approach were more dispersed, whereas it exhibited good consistency with the measurement data in terms of the on-off curves and few-data situations.

- The paper by Zhou et al. (2021a) applied lattice Boltzmann method (LBM) on six types of fibrous filter media with lognormal distribution models. They found that the resistance was relatively low when the solid fraction inside the filter media was uniformly distributed, while the filter media with a random lognormal-distribution model demonstrated the best filtration performance in terms of quality factor. The study provides a viable numerical method for the performance optimization of air-filtration devices for the next-generation cleanroom industry.

- The paper by Ye et al. (2021) explored air curtains equipped on normal consulting desk to prevent doctors' direct exposure to patient exhaled pollutants. The average mass fraction of exhaled pollutants decreased significantly in the consulting ward with air curtains through numerical simulations. Both the velocity and angle of the supply air have great effects on the cutting-off performance. This study can provide effective and intuitive guidance for applying air curtains in consulting wards.

- The paper by Zhuang et al. (2021) used the computational fluid dynamics (CFD) method to investigate the indoor environment and thermal comfort under the all air system (AAs) and the radiant floor cooling and wall cooling combined with air supply system (RC/ASs) in a train station waiting hall. The results showed that indoor air distribution in most areas under the RC/ASs was more uniform based on the indoor heat and humidity requirements under the cooling load of the measured day. Meanwhile, the RC/ASs addressed the problem that the temperature around the seated passengers in the waiting area was relatively high in the AAs.

- The paper by Tong et al. (2021) proposed a novel approach to map the urban natural ventilation potential based on the similarity between water flows and air flows. By integrating the urban terrain/form model and prevailing wind pressure model, an air flow digital elevation model (AF-DEM), which represents the resistance to airflow and can be used for hydrological simulation, is generated and applied to evaluate the urban airflow patterns. Taking Nanjing as a case study, 8 AF-DEMs based on different wind direction/speed conditions were generated, and their corresponding ventilation corridor maps were constructed.

- The paper by Zhang et al. (2021) proposed a CFD model along with a dimensionless quantitative assessment standard-the air pollution residual time (APRT) to evaluate the local haze-fog dispersion in a built environment. The most favorable orientation of the studied building group can reduce APRT by more than $50 \%$. Their results suggested that in order to reduce the consequential negative effects of air pollution, future urban designs should undergo a comprehensive ventilation assessment to ensure a low APRT value.

- The paper by You and Ding (2021) used CFD simulation techniques to investigate the effects of urban square entry layouts on spatial ventilation under different surrounding building conditions. They found that square street entry layouts could have an obvious effect on spatial ventilation under low surrounding building densities. In addition, when the wind direction is perpendicular to the square street entry, the corner oblique-entry layout square shows a better ventilation performance. When the wind direction is oblique to the square street entry, the lateral-entry layout design shows the best ventilation performance.

- The paper by Ming et al. (2021) investigated the effects of wind catcher, wind speed and the geometry of the street canyons on pollutant dispersion by using CFD simulation. Their results showed that adding wind catchers can significantly improve the air quality of step-down street canyons and reduce the average pollutant concentrations in street canyons by $75 \%$. Higher wind speed enhances the diffusion of pollutants differently in different geometric street canyons.

In addition, we would also like to draw the readers' attention to several important key-note lectures delivered at CHAMPS 2019, including:

- "Indoor air quality design and control in low energy residential buildings" by Professor Carsten Rode (Technical University of Denmark, Denmark) and Professor John Grunewald (Dresden University of Technology, Germany). They presented a deep and detailed discussion on how to provide a comfortable and healthy indoor environment while saving energy in future residential buildings, and sought to find an ideal balance between energy efficiency and ventilation demand in the consideration of indoor pollutants.

- "Knowing ahead: using simulation to prevent indoor air pollution" by Professor Xudong Yang (Tsinghua University, China). He discussed the related problems due to indoor air pollution, simulation and validation approaches to indoor pollution sources and sinks, and prevention methods by taking formaldehyde and VOCs as examples.

- "Creating outdoor thermal comfort via sub microclimate engineering approach" by Professor Jianlei Niu (Hong Kong Polytechnic University, Hong Kong, China). He introduced how to create local cooling points of outdoor and semioutdoor environments and the impact of building blocks on outdoor thermal comfort in warm urban environment.

- "Demonstration projects: ventilation performance of 60 residential buildings over 5 climate zones of China" by 
Dr. Jun Ren (Shenzhen Institute of Building Research Co. Ltd., China). He introduced 60 building ventilation and air purification demonstration projects to conduct long-term monitoring and investigation to study the ventilation performance and indoor air quality.

- "Wind and thermal environment at an urban neighborhood scale-a quantitative study on vegetated urban microclimate" by Professor Naiping Gao (Tongji University, China). He discussed the interaction between urban vegetation and urban climate at different scales through the exchange of momentum, energy and water vapor, with an emphasis on the roles of trees in air pollutant diffusion, air quality and thermal comfort at a typical neighborhood scale.

Future CHAMPS forums will continue to focus on major challenges facing the combined heat, air, moisture and pollutant simulations for the design and operation of sustainable buildings; highlight the most recent progress; and identify opportunities for further collaboration in CHAMPS research, development and applications. Topics may include:

- Whole building indoor environmental quality (IEQ) and energy performance: modeling and simulation of combined heat, air, moisture, and pollutant transport processes in and around buildings, and the impact of processes on IEQ and building energy efficiency;

- Healthy and intelligent buildings: interaction and interdependencies between IEQ and energy efficiency measures, effectiveness of source reduction, ventilation and air cleaning strategies; model-based predictive control, and application of Internet of Things (IOTs) technology in distributed building environmental control;

- Climate, community and site planning: climate change effects on IEQ, urban and community planning, microurban climate and energy efficiency of building clusters;

- Building envelope performance: hygrothermal performance of buildings in different climates, leakage and moisture control, envelope-integrated ventilation and energy storage systems, energy and durability;

- Micro-environment around occupants: demand-based personal environmental control, occupant behavior and impacts on IEQ and energy efficiency;

- Design studio: methods and tools for coordinated and integrated urban and building systems design, building information modeling (BIM), CHAMPS simulation framework, software, and optimization techniques, common databases of materials, assemblies, building topologies, climates, and real-world versus design performances;
- Case studies: applications of CHAMPS in building systems design and model-based predictive controls;

- Underground space environment: modeling and simulation of wall surface heat flow and thermal environment, ventilation, pollutant transport and distribution in underground and semi-underground spaces;

- Development and applications of CHAMPS tools to predict the spread of infectious diseases such as COVID-19 and analyze the effectiveness of various strategies for reducing the risk of infection due to airborne transmission.

We look forward to furthering the scientific exchange and international collaborations.

\section{References}

Liu Z, Nicolai A, Abadie M, et al. (2021). Development of a procedure for estimating the parameters of mechanistic VOC emission source models from chamber testing data. Building Simulation, 14: 269-282.

Ming T, Han H, Zhao Z, et al. (2021). Field synergy analysis of pollutant dispersion in street canyons and its optimization by adding wind catchers. Building Simulation, 14: 391-405.

Shen J, Krietemeyer B, Bartosh A, et al. (2021). Green Design Studio: a modular-based approach for high-performance building design. Building Simulation, 14: 241-268.

Tong Z, Luo Y, Zhou J (2021). Mapping the urban natural ventilation potential by hydrological simulation, Building Simulation, 14: 351-364.

Ye J, Qian H, Ma J, et al. (2021). Using air curtains to reduce shortrange infection risk in consulting ward: a numerical investigation. Building Simulation, 14: 325-335.

You W, Ding W (2021). Effects of urban square entry layouts on spatial ventilation under different surrounding building conditions. Building Simulation, 14: 377-390.

Zhang Y, Yu Y, Kwok KCS, et al. (2021). CFD-Based Analysis of Urban Haze-Fog Dispersion-A Preliminary Study, Building Simulation, 14: 365-375.

Zhao J, Zhang J, Grunewald J, et al. (2021). A probabilistic-based method to evaluate hygrothermal performance of an internally insulated brick wall. Building Simulation, 14: 283-299.

Zhou B, Wu X, Chen L, et al. (2021a). Modeling the performance of air filters for cleanrooms using lattice Boltzmann method. Building Simulation, 14: 317-324.

Zhou X, Liu T, Yan D, et al. (2021b). An action-based Markov chain modeling approach for predicting the window operating behavior in office spaces, Building Simulation, 14: 301-315.

Zhuang B, Shi J, Chen Z (2021). Numerical Study on indoor environment and thermal comfort in train station waiting hall with two different air-conditioning modes, Building Simulation, 14: $377-345$. 